\title{
WestVirginiaUniversity
}

THE RESEARCH REPOSITORY @ WVU

Graduate Theses, Dissertations, and Problem Reports

1998

\section{Resonant ion heating in a helicon plasma}

John L. Kline

West Virginia University

Follow this and additional works at: https://researchrepository.wvu.edu/etd

\section{Recommended Citation}

Kline, John L., "Resonant ion heating in a helicon plasma" (1998). Graduate Theses, Dissertations, and Problem Reports. 920.

https://researchrepository.wvu.edu/etd/920

This Thesis is protected by copyright and/or related rights. It has been brought to you by the The Research Repository @ WVU with permission from the rights-holder(s). You are free to use this Thesis in any way that is permitted by the copyright and related rights legislation that applies to your use. For other uses you must obtain permission from the rights-holder(s) directly, unless additional rights are indicated by a Creative Commons license in the record and/ or on the work itself. This Thesis has been accepted for inclusion in WVU Graduate Theses, Dissertations, and Problem Reports collection by an authorized administrator of The Research Repository @ WVU. For more information, please contact researchrepository@mail.wvu.edu. 


\title{
Resonant Ion Heating in a Helicon Plasma
}

\author{
John L. Kline \\ Thesis submitted to the College of Arts and Sciences of \\ West Virginia University \\ In partial fulfillment of the requirements for the degree of \\ Master of Science \\ in \\ Physics
}

\author{
Earl E. Scime, Chair \\ Mark E. Koepke \\ John E. Littleton
}

Fall 1998

Morgantown, West Virginia 


\begin{abstract}
A resonant ion heating system has been developed for heating ions and controlling the ion temperature anisotropy in a helicon plasma source. The system uses two rectangular coils placed on either side of the cylindrical pyrex vacuum chamber to create a time dependent magnetic field transverse to the steady state axial field. A $1 \mathrm{~kW}, 25-125 \mathrm{kHz} \mathrm{RF}$ generator powers the coils. The parallel and perpendicular ion temperatures in argon plasmas are measured with a laser induced fluorescence diagnostic tuned to a metastable argon ion transition. Ion heating of over $800 \%$ has been measured for the perpendicular ion temperatures with an increase of $70 \%$ in the parallel direction. This temperature increase occurs for a specific ratio of heating frequency to ion cyclotron frequency. Evidence suggesting that ion Bernstein waves are responsible for the ion heating is presented.
\end{abstract}




\section{Table Of Contents}

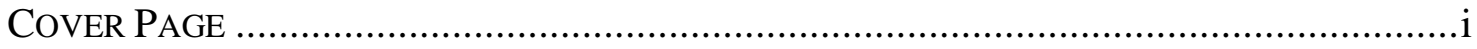

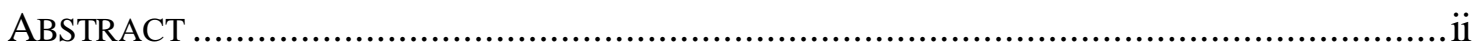

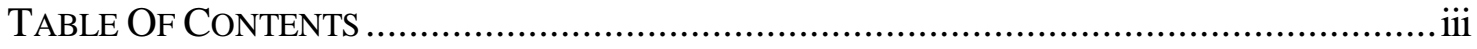

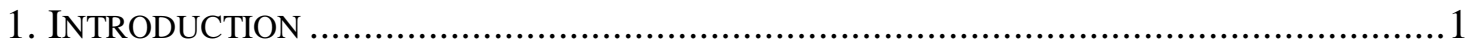

2. LEIA AND HELIX PARAMETERS, DIAGNOSTICS, AND CHARACTERISTICS ................. 3

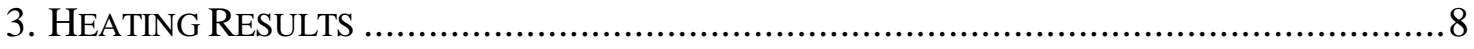

$3.1 \mathrm{~m}=0$ coil and Stix coil antenna .......................................................... 9

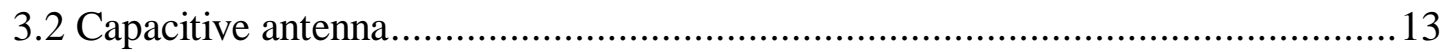

3.3 Circular Transverse and Rectangular Transverse Inductive Antennas ................. 14

3.4 Transverse Inductive Plus Capacitive Antenna ...........................................20

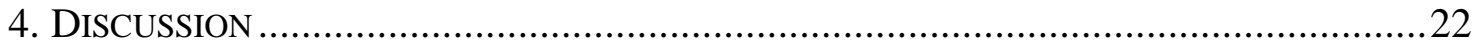

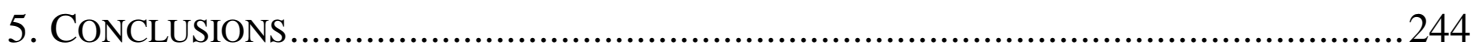

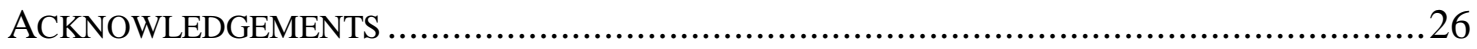

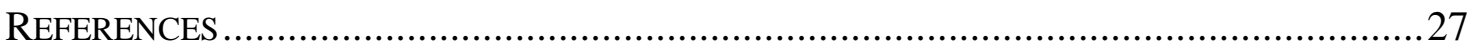




\section{Introduction}

The Large Experiment on Instabilities and Anisotropies (LEIA) was constructed to study space plasma phenomena in the laboratory. The plasma for LEIA is generated with the Hot hELIcon eXperiment (HELIX) inductively coupled plasma source [1]. With an appropriate choice of HELIX and LEIA operating parameters, high $\beta_{e}$ $\left(\beta=n k T_{e} / B^{2} \approx 1\right)$, steady state, fully magnetized plasmas are created in LEIA. One of the objectives of LEIA is to examine ion temperature anisotropy driven instabilities. Anisotropy is defined as $T_{\perp} / T_{\| /}$, where parallel and perpendicular refer the orientation with respect to the ambient magnetic field. To excite these instabilities, a significant temperature anisotropy $\left(T_{\perp} / T_{\|}>2\right.$ or $\left.T_{\perp} T_{\|}<.5\right)$ at high $\beta_{i}, \beta_{i} \approx .1$, is required [2]. Previous experiments [3] demonstrated that HELIX has an intrinsic temperature anisotropy that depends strongly on the source magnetic field strength (Figure 1), and weakly on other source parameters such as neutral pressure and RF power. For the instability experiments, separate control of the ion temperature anisotropy and magnetic field strength is desirable. Therefore, an auxiliary ion heating system for HELIX has been constructed using an extremely low frequency (ELF), $10 \mathrm{kHz}<f<100 \mathrm{kHz}$, generator, and various antennas.

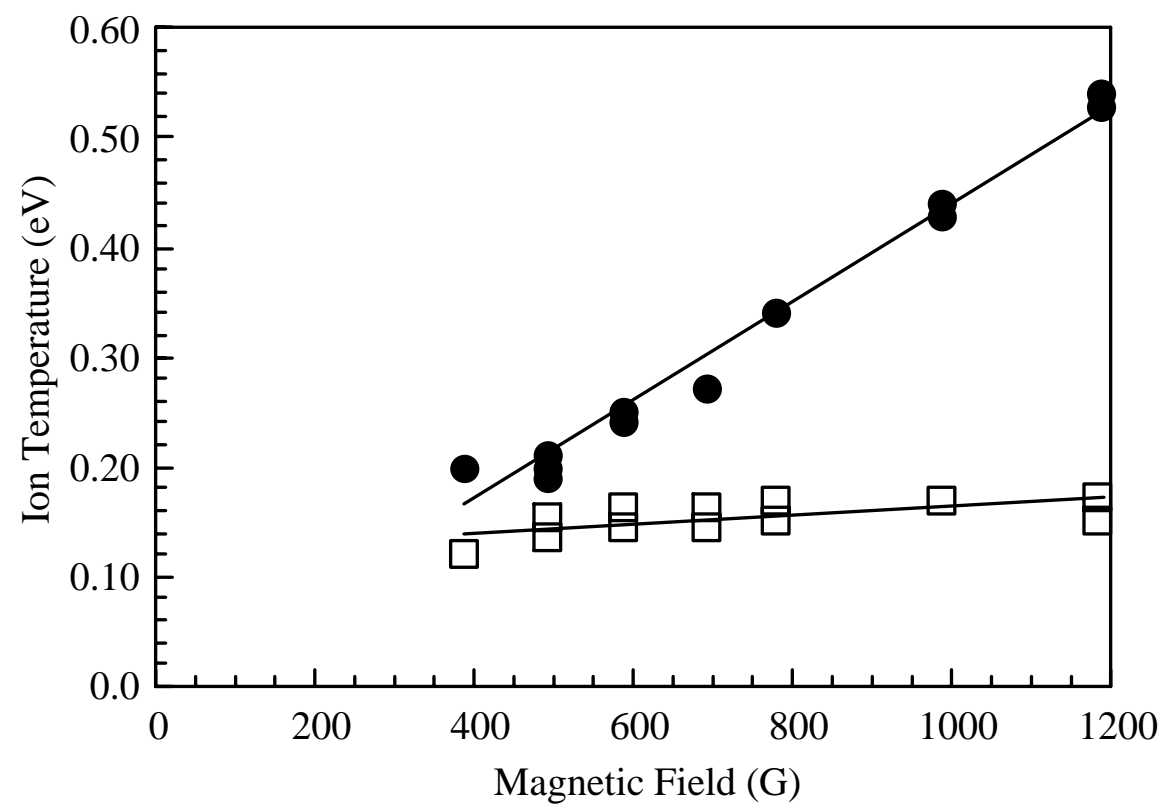

Figure 1. The perpendicular ion temperature in HELIX increases with magnetic field while the parallel ion temperature stays constant, creating an intrinsic temperature anisotropy. 
Since the beginnings of plasma physics, ion heating systems have been developed for both fusion and small-scale laboratory experiments, e.g., tokamaks [4], mirror machines [5], stellarators [6], and Q-machines [7,8]. Although ion heating through collisions with heated electrons [9] or neutral beams [10] has been used, a wave-particle resonance is typically exploited to directly heat the ions. A variety of wave-particle resonances have been investigated, e.g., ion cyclotron [4,6,7,8], lower hybrid [9], Alfven [9], upper hybrid [11], and ion Bernstein [12], with ion cyclotron resonant heating being the most common in tokamaks and linear devices $[4,7,8,9,10]$. The greatest technical challenge to ion cyclotron resonant heating (ICRH) in a helicon source arises from the helicon source being a low ion temperature, high-density plasma confined by a modest magnetic field. With of $T_{i} \sim 0.3 \mathrm{eV}, n \sim 1.0 \times 10^{13} \mathrm{~cm}^{-3}$, and $B_{o} \sim 1 \mathrm{kG}$, these conditions produce a large ratio of ion collision frequency to ion gyro frequency, $2 \pi v_{i i}, \Omega_{i} \sim 120$, which does not allow the ions to complete a significant portion of a gyro orbit. In a Q-machine, Good et al. [7] used a Nagoya type III antenna for ICRH in a low density barium plasma, $n \sim 5 \times 10^{9} \mathrm{~cm}^{-3}$, confined by a modest magnetic field, $B_{o}=1.6 \mathrm{kG}$. For those experiments, the low ion cyclotron frequency $\Omega_{i} \sim 207 \mathrm{krad} / \mathrm{s}$, did not prevent ICRH because the low density reduced the collision frequency enough that $2 \pi v_{i i}, \Omega_{i} \sim 0.06$. For fusion devices like tokamaks, a strip antenna [9] can be used to launch a wave at the cyclotron frequency to heat the ions. The high temperatures and large magnetic fields of a tokamak also keep the ratio of the collision frequency to the gyro frequency small. With typical tokamak operating parameters [10] of $T_{i} \sim 22 \mathrm{keV}$, $n \sim 0.81 \times 10^{20} \mathrm{~m}^{-3}$, and $B_{o} \sim 11$ Tesla, $2 \pi v_{i i} \Omega_{i}$ is approximately $2.5 \times 10^{-7}$. It is the value of $2 \pi v_{i i} \Omega_{i} \sim 120$ that distinguishes the helicon source from other high density plasma devices.

In this paper, we present ion heating results for six different heating antennas in a helicon source. The electromagnetic field from each of these antennas provides a source of free energy to HELIX, in addition to the electromagnetic field from the helical driving antenna that creates the plasma. The objective of the experiment was to determine the optimum antenna configuration and driving frequency for heating the ions. In section 2, we present the experimental parameters and diagnostics. In section 3, we present the 
results and a discussion for each antenna. In section 4, we review the results and discuss possible mechanisms to explain the observed heating.

\section{LEIA and HELIX parameters, diagnostics, and characteristics}

HELIX (Figure 2) consists of a Pyrex tube $15 \mathrm{~cm}$ in diameter and $157 \mathrm{~cm}$ long. Two pairs of opposing $2.5 \mathrm{~cm}$ ports in a cross formation located $52 \mathrm{~cm}$ from one end are used for Laser Induced Fluorescence (LIF) measurements. Ten water cooled electromagnets produce an axial magnetic field of 0 - 1300 Gauss. The source gas is argon at neutral pressures of 1 to 10 mTorr. RF power of up to $2.5 \mathrm{~kW}$ over a frequency range of $6-20 \mathrm{MHz}$ is used to create the plasma. The frequency range is limited to $6-20 \mathrm{MHz}$ by the Pi matching circuit. The driving antenna is a $19 \mathrm{~cm}$ long, half wave, right-handed helix made from $2 \mathrm{~cm}$ wide copper strips [13]. The right-handedness is relative to the magnetic field direction, and is designed to launch the $m=+1$ helicon wave towards the LEIA chamber.

\section{LEIA}

\section{HELIX}
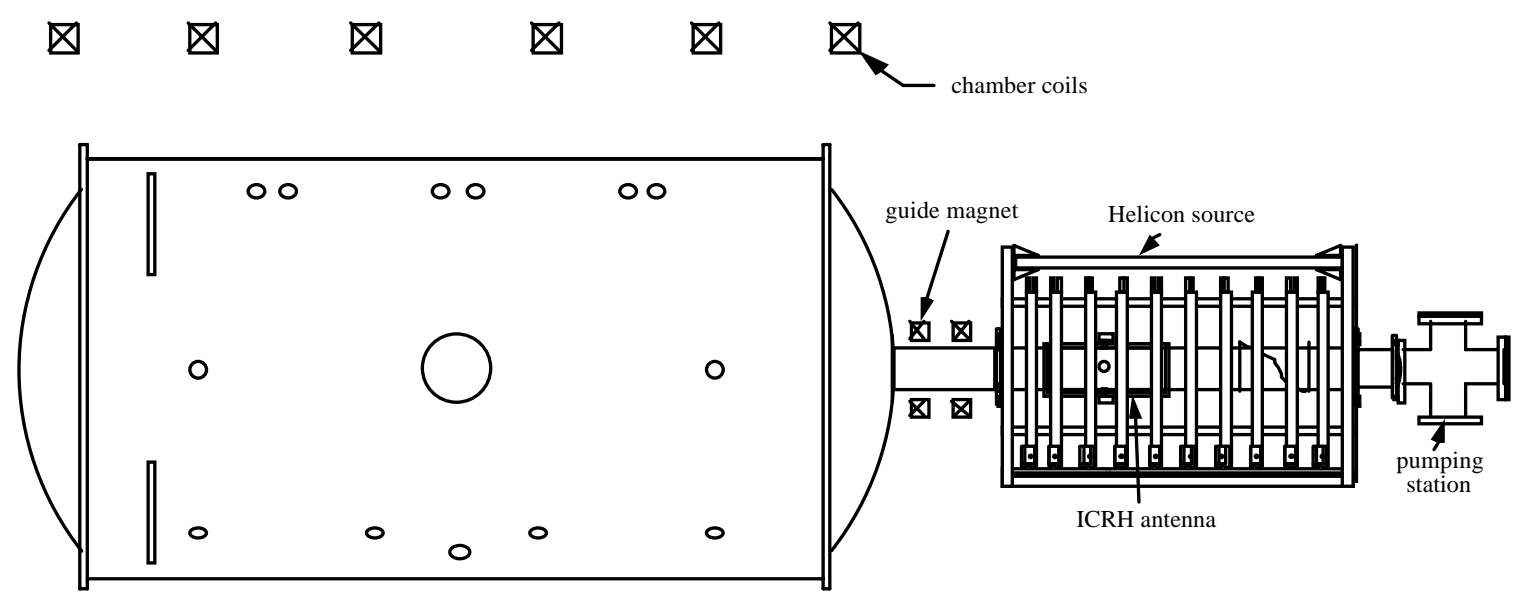

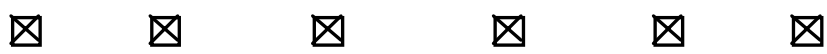

Figure 2. The helicon source, HELIX, connected to LEIA.

Typical electron temperature and densities in HELIX are $T_{e} \sim 5 \mathrm{eV}$ and $n \sim 1 \times 10^{13} \mathrm{~cm}^{3}$ for a RF power of $2.0 \mathrm{~kW}$ and neutral pressure of $3.7 \mathrm{mTorr}$. At the end 
opposite the driving antenna, HELIX is connected to LEIA through an 6.5" O. D. stainless steel bellows (Figure 2).

LEIA is a large aluminum chamber $2 \mathrm{~m}$ in diameter and $4.5 \mathrm{~m}$ long. Seven, 2.75 m diameter, water cooled electromagnets surround the chamber. The axial magnetic field in LEIA can be varied from 0-80 Gauss with a spatial ripple of less than $1 \%$ along the axis. For all the experiments reported here, the magnetic field in LEIA was fixed at 18 Gauss. The base pressure in the combined LEIA and HELIX system is $8 \times 10^{-8}$ Torr.

The primary diagnostic for these experiments is laser induced fluorescence (LIF) [14]. The absorption and emission frequencies of an atom or ion moving relative to a radiation source are Doppler shifted by an amount proportional to the component of velocity along the radiation direction. During a typical LIF measurement, the frequency of a narrow bandwidth laser is swept across a collection of ions or atoms that have a thermally broadened velocity distribution. The absorption spectrum for the entire ensemble of atoms or ions has associated with it a width and a shift from the natural frequency that is used to determine the temperature and flow velocity of the particle distribution. The LIF system used in these experiments consists of a $6 \mathrm{~W}$ Coherent Innova 300 argon-ion laser that pumps a Coherent 899 ring dye laser. A beam splitter diverts $10 \%$ of the dye laser's light into a Burleigh 1500 wavemeter to monitor the wavelength. The wavemeter has a resolution of $0.0001 \mathrm{~nm}$. For argon plasmas, the output of the dye laser is tuned to the $611.492 \mathrm{~nm}$ line to match the $3 \mathrm{~d}^{2} \mathrm{G}_{9 / 2}$ to $4 \mathrm{p}^{2} \mathrm{~F}_{7 / 2}$ transition of singly ionized argon ions. As the dye laser performs a $10 \mathrm{GHz}$ sweep, corresponding to a wavelength sweep of 0.012 $\mathrm{nm}$, the fluorescent emission from the upper metastable level (see Figure 3) is measured with a filtered photomultiplier tube detector.

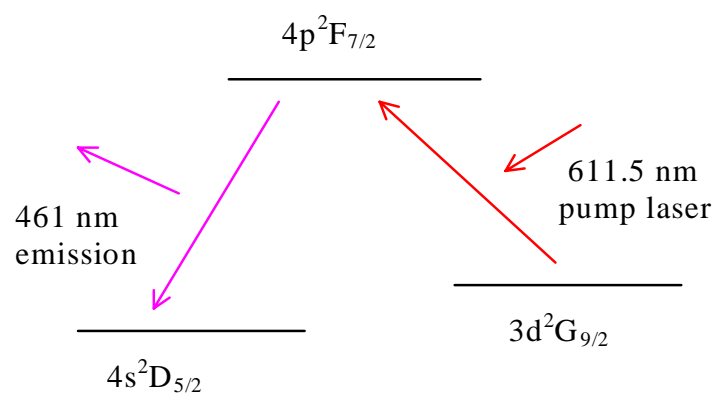

Figure 3. Argon LIF scheme used for ion temperature measurements. 
The filter in front of the photomultiplier has a $1.0 \mathrm{~nm}$ passband centered around the emission line $(461 \mathrm{~nm})$. The output of the dye laser is chopped at $1 \mathrm{kHz}$. The chopping signal is used as the reference for a Stanford Research SR830 lock-in amplifier that monitors the photomultiplier tube signal and distinguishes the fluorescence signal from the intense background emission at the same wavelength using phase synchronous detection. The amplified signal from the SR830 is sent to a Tektronics VXI 641 digital oscilloscope. A Pentium based microcomputer retrieves the LIF signal, which is fitted to a single Gaussian distribution to determine the ion temperature and the center frequency.

Perpendicular and parallel components of the argon ion temperature can be obtained by injecting the laser light into the plasma perpendicular and parallel to the magnetic field, respectively, and collecting the emitted light perpendicularly to the injected beam (Figure 4). The argon absorption line includes a number of component distinguishable by Zeeman-splitting. For perpendicular measurements, only the linearly polarized $\pi$ transitions are excited. For the magnetic field strengths in HELIX, the Zeeman splitting for these lines is on the order of $2.6 \times 10^{-4} \mathrm{~nm}$ at 1300 Gauss, compared to the Doppler-broadened line width of $2.6 \times 10^{-3} \mathrm{~nm}$, and can be ignored. For the parallel measurements, two circularly polarized $\sigma$ transitions are excited. Since the Zeeman splitting of the $\sigma$ lines is on the order of the thermal broadening, Zeeman splitting for parallel measurements cannot be ignored. Because the laser light is transported through a multimode fiber, the polarization of the laser is not preserved. By introducing a combination of a linear polarizer followed by a quarter wave plate into the parallel injection optics, a single circular polarization is selected and only one of the two $\sigma$ transitions is detected. The additional optical components also reduce the overall intensity of the laser light and lower the signal-to-noise ratio for parallel ion temperature measurements.

For both $T_{i \perp}$ and $T_{i \|}$ measurements, there are a number of possible sources of systematic error including Zeeman splitting $\left(2.6 \times 10^{-4} \mathrm{~nm}\right)$. Other sources of systematic error include Stark broadening $\left(1.2 \times 10^{-6} \mathrm{~nm}\right)$, the line width of the laser $\left(1.3 \times 10^{-6} \mathrm{~nm}\right)$, and the natural line width of the argon transition $\left(2.3 \times 10^{-5} \mathrm{~nm}\right)$. Zeeman splitting is the 
largest, and limits the absolute temperature measurement to approximately $0.04 \mathrm{eV}$ at high magnetic fields. Since the measurements reported here will compare two ion temperature measurements with the same source parameters, the impact of systematic errors is minimal.

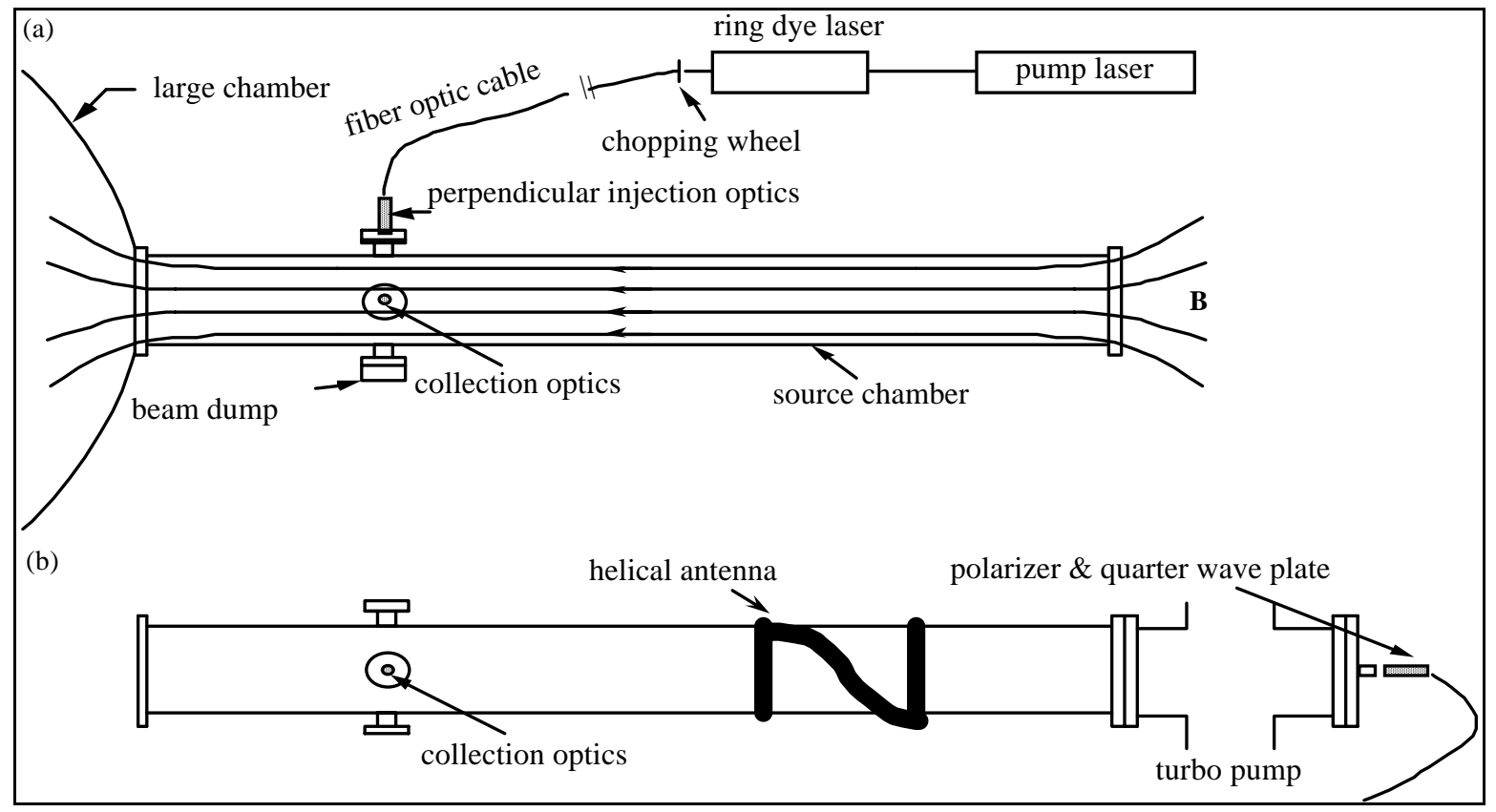

Figure 4. LIF diagnostic setup used to measure the ion velocity distribution both (a) perpendicular and (b) parallel to the axial magnetic field.

Random errors due to fluctuations in the plasma and laser power fluctuations are also a concern. To estimate the effect of the random errors, several measurements with the heating circuit on and off in both the perpendicular and parallel directions were taken with all other parameters held constant. The error was less than $3 \%$ for all $T_{i \perp}$ and $T_{i \|}$ measurements made with the heating circuit off. With the heating circuit on, the error was less than 3\% for measurements made at a neutral pressure of 3.0 mTorr. At a neutral pressure of 1.5 mTorr, the error approached $15 \%$ because the distribution width was nearly as wide as the laser scan width. In addition to systematic and random errors, LIF measurements in RF generated plasmas are prone to errors arising from discrepancies between the measured distribution and the actual distribution. The effect of ion "sloshing" on the distribution is well known [15]. 
Ion "sloshing" arises from oscillatory motion of the ions along the path of the laser due to a time dependent force from the wave field. Such motion could systematically widen the ion velocity distribution. To confirm the absence of ion sloshing effects in the ion temperature measurements, the decay rate of ion temperature was compared to the decay of the heating circuit. With the laser fixed at $+2 \mathrm{GHz}$ relative to the peak of the absorption profile, the heating circuit was turned off. The 1/e decay time for the ratio of the $+2 \mathrm{GHz}$ signal to the peak signal from the heating on case to the heating off case was observed to be approximately $0.7 \mathrm{~s}$. The decay coefficient for the RLC heating circuit was measured to be approximately $0.003 \mathrm{~s}$. Thus the ion temperature increase persists considerably longer than the time the antenna circuit is powered and the measured temperatures are not an artifact of ion sloshing.

The ion heating system was designed to operate at the ion cyclotron frequency or its second harmonic to exploit the ion cyclotron resonance. For the range of magnetic field strengths in HELIX, the cyclotron frequency of an argon ion is in the range of 15 - $50 \mathrm{kHz}$. An ENI Plasmatek 1, 20 - $125 \mathrm{kHz}, 1000$ Watt RF generator with a $50 \mathrm{ohm}$ output impedance is used as the RF source. To match the antennas to the RF generator, six large capacitors were combined to form an L network matching circuit (Figure 5). The $\mathrm{L}$ network converts the inductive antenna load into a real impedance with capacitor values of $\mathrm{C}_{1}=6.8 \mu \mathrm{F}$ and $\mathrm{C}_{2}=19.6 \mu \mathrm{F}$. The resulting real impedance for the antenna matching circuit is less than $1 \mathrm{ohm}$, which limits the amount of power that can be delivered to the circuit because the internal current limit of the RF generator is reached well before its power limit. Six different antenna configurations were attached individually to the RF generator for these experiments and each configuration was operated at similar power levels. Since fixed capacitors were used, the HELIX magnetic field was varied with the RF frequency held fixed to look for the optimum magnetic field for ion heating with each antenna. 


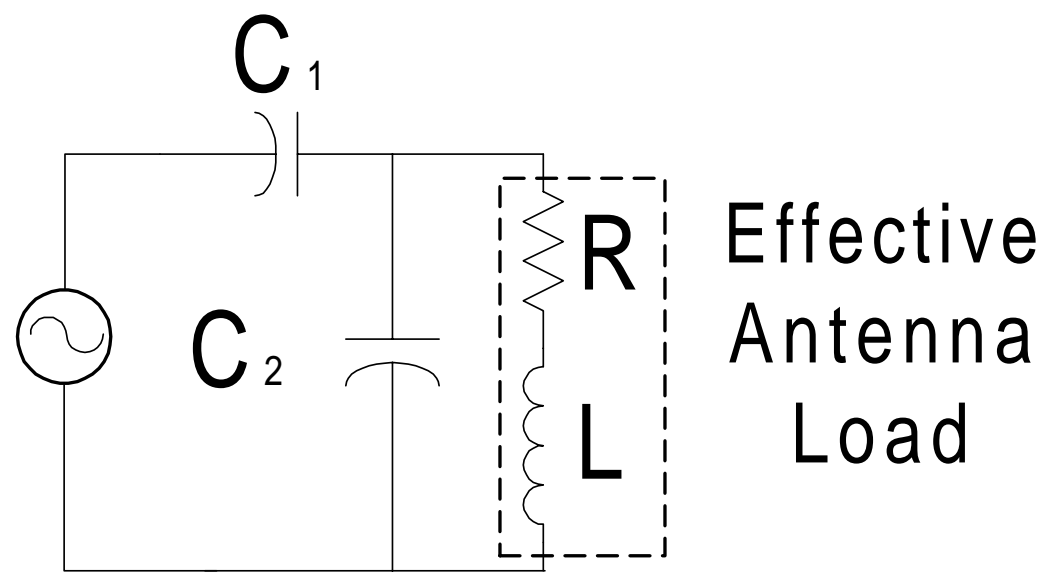

Figure 5. L matching circuit used in ion heating experiments.

\section{Heating Results}

At each magnetic field strength, the perpendicular ion temperature was measured with the heating power on and with the heating power off to determine the heating efficiency. Heating efficiency is the percent ion temperature increase with the heating power on compared to the ion temperature with the heating power off:

$$
\text { Heating Efficiency }=\frac{T_{i(o n)}-T_{i(o f f)}}{T_{i(o f f)}}
$$

If the perpendicular results appeared promising, additional parallel ion temperature measurements were made and then both perpendicular and parallel ion temperatures were measured at a second neutral pressure. Experimental data for six antenna configurations are presented. For the $\mathrm{m}=0$ coil, Stix coil, Capacitive, Circular Transverse, and the Circular Transverse plus Capacitive antennas, the neutral pressure was 3.0 mTorr and the heating power was 550 Watts. For the Rectangular Transverse antenna, the neutral pressures were 1.5 mTorr and 3.0 mTorr and the heating power was 200 Watts. 


\section{$3.1 \mathrm{~m}=0$ coil and Stix coil antenna}

The $\mathrm{m}=0$ coil and Stix Coil [6] antenna consist of loops of 12 gauge wire wrapped azimuthally around the plasma chamber. Both coils are inductive, and designed to generate electric fields at the cyclotron frequency to resonate with the ion cyclotron motion. The $\mathrm{m}=0$ coil (Figure 6a) creates an oscillatory magnetic perturbation along the axial background magnetic field. According to Faraday's law, this time dependent magnetic perturbation creates a circular electric field perpendicular to the magnetic field. This electric field oscillates between the clockwise and the counter clockwise direction, accelerating or decelerating the ions based on their position in the gyro orbit (Figure 7). Within a few gyro periods, the ions' position in their gyro orbit are in phase with the electric field such that when the electric field is maximum in each direction (clockwise or counter clockwise) the velocity vector and the electric field vector are aligned. In other words, the ion motion is resonant with the electric field and the perpendicular ion velocities increase. Collisions between the ions thermalize this additional kinetic energy into temperature.

For a confined plasma, a single $\mathrm{m}=0$ coil is not expected to work [6]. As the ions gain energy, their gyro orbits increase in size. The density gradient inherent in a confined plasma leads to a net radial charge imbalance and an oscillating space charge radial electric field. The additional radial electric field changes the direction of the net electric field, and prevents the ion motion from resonating with the azimuthal electric field. The radial electric field can be eliminated by the use of a Stix coil antenna [16] (Figure 6b). The current in each coil travels in the opposite direction; thus the time dependent electric fields created by each coil are 180 degrees out of phase. In this configuration (Figure 8), the ions affected by each coil are also 180 degree out of phase, creating a space charge electric field parallel to the magnetic field. Electrons then travel along the magnetic field and cancel the radial component of the electric field near each coil leaving only the azimuthal electric field induced by each coil. Without the space-charge radial electric field, the ions can be accelerated by the azimuthal electric field and the additional kinetic energy thermalized through collisions. 
a)
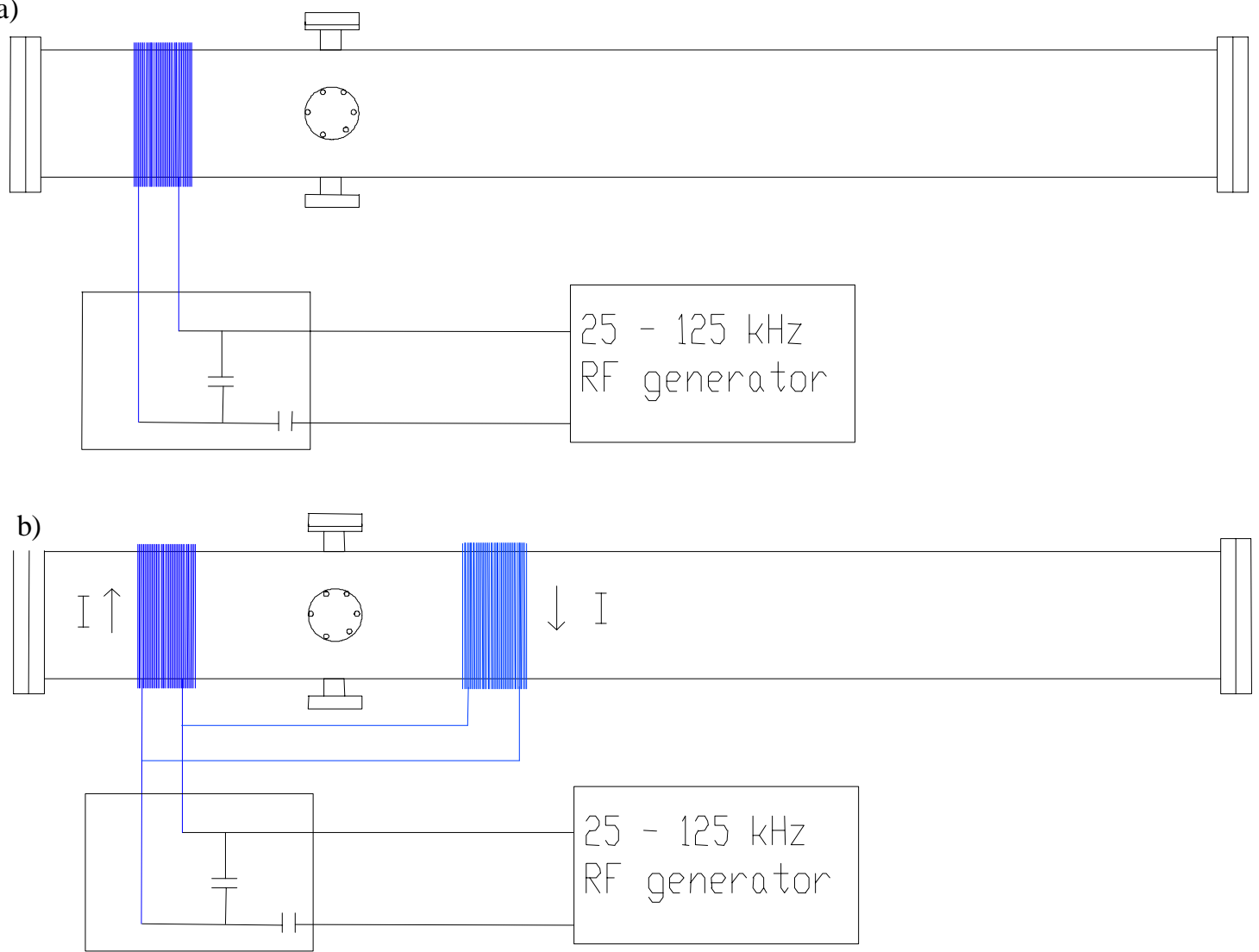

Figure 6. a) $\mathrm{m}=0$ Coil antenna b) Stix Coil [6] antenna. Both antennas are shown with the glass chamber and RF generator.

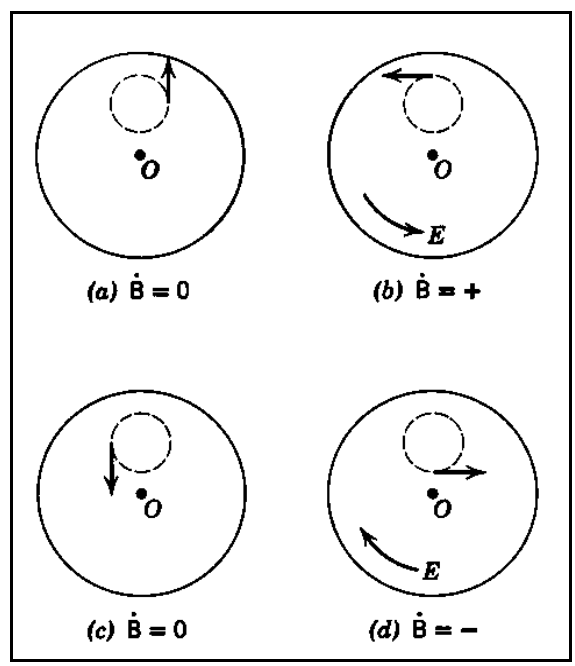

Figure 7. This figure, as shown in Rose and Clark [6], shows four phases of the time dependent magnetic field due to a azimuthally wrapped loop antenna, and the position of an ion in its orbit relative to the center of the plasma, $\mathrm{O}$. The background magnetic field points out of the page. 


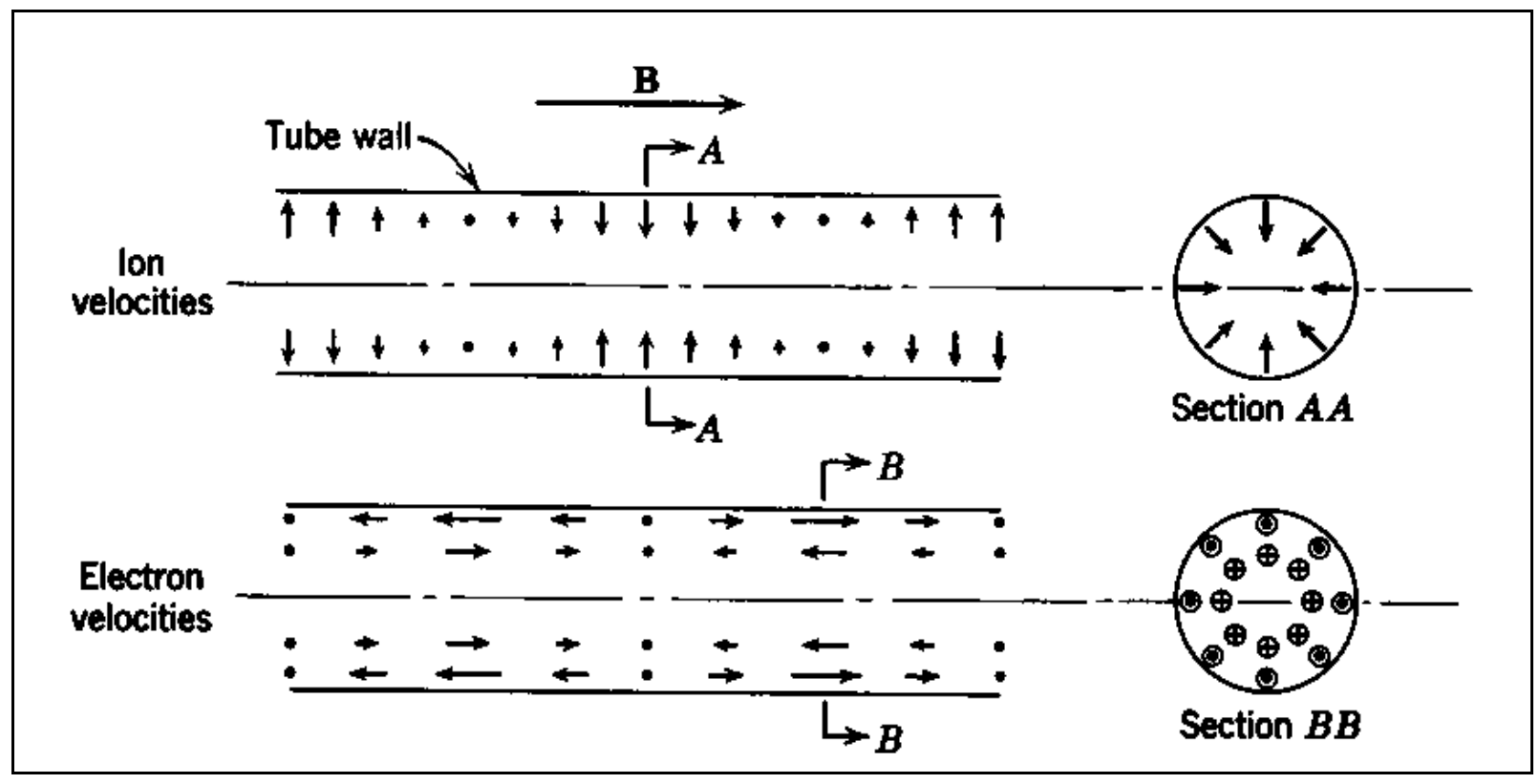

Figure 8. The electron and ion velocities with the Stix coil as shown by Rose and Clark [6].

Operation of both the $\mathrm{m}=0$ coil and the Stix coil antenna at a neutral pressure of 3.0 mTorr did not show any evidence of ion heating in HELIX (Figure 9). A possible explanation for the lack of heating with these antennas is that phase bunching of the ions is required to convert the additional kinetic energy from the electric field into thermal energy. Because the ion cyclotron frequency, $f_{\mathrm{ci}} \sim 30 \mathrm{kHz}$, is so much smaller than the ion-ion collision frequency, $v_{i i} \sim 8 \mathrm{MHz}$, it is impossible for any of the ions to complete a significant portion of a gyro-orbit, much less get "in phase" with the electric field and gain kinetic energy. 
a)

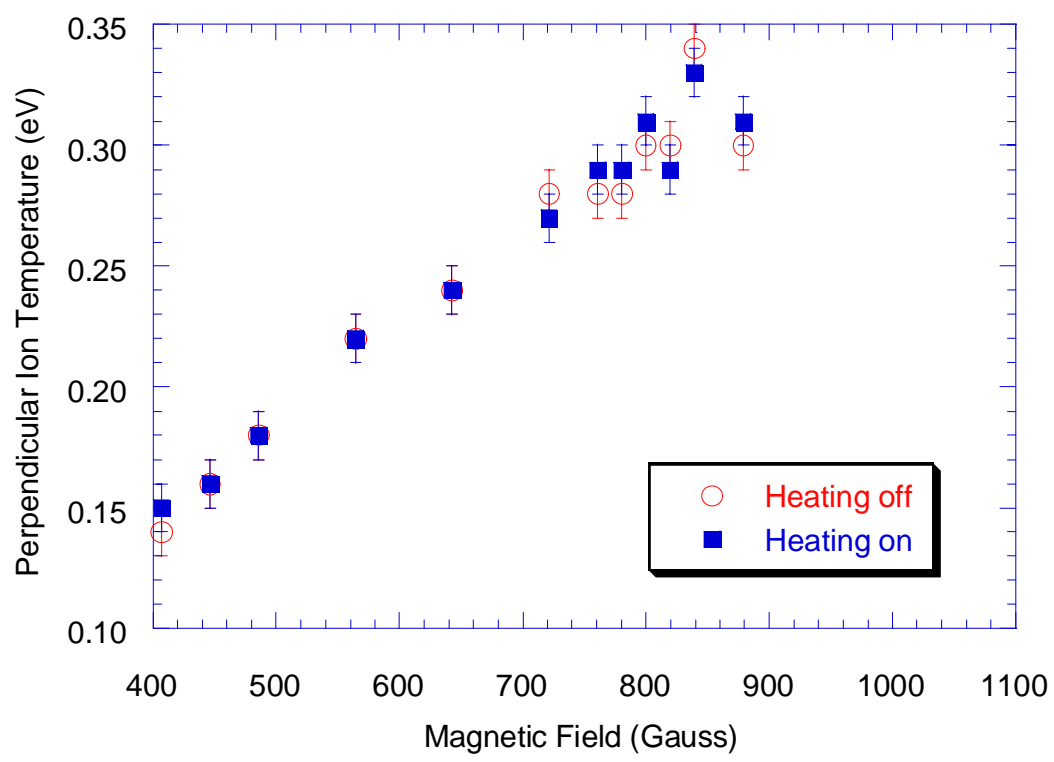

b)

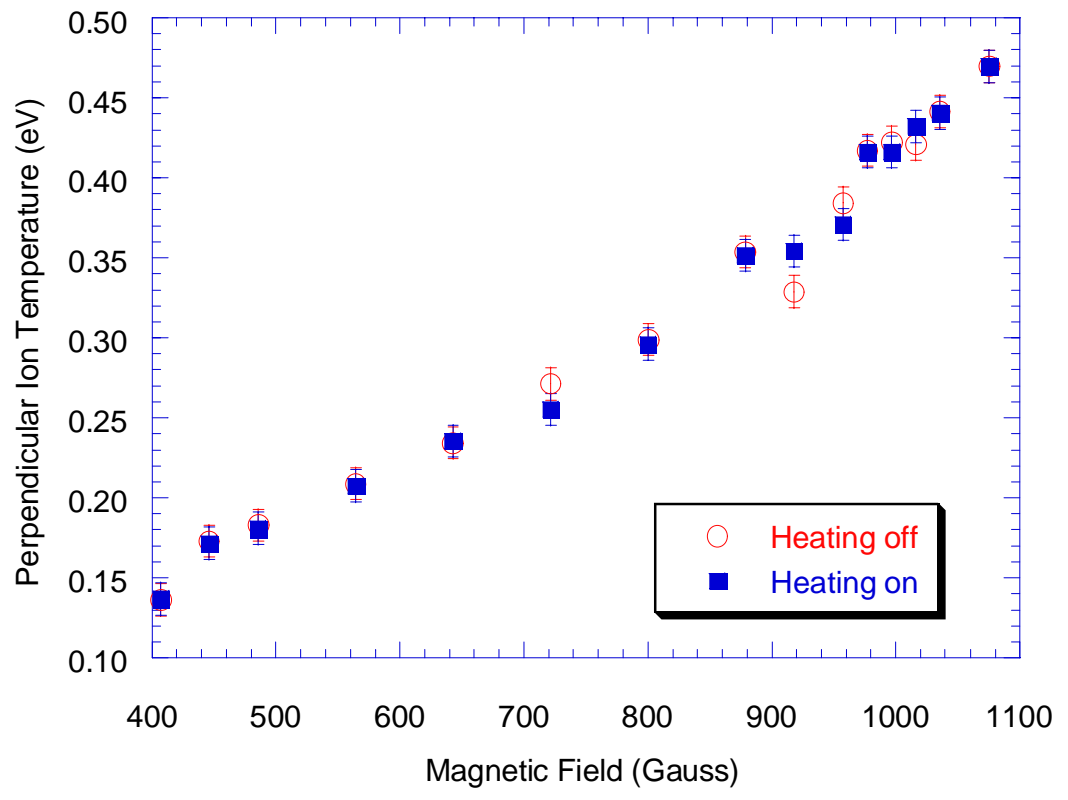

Figure 9. a) Perpendicular ion temperature verses magnetic field for the $m=0$ mode coil antenna. b) Perpendicular ion temperature verses magnetic field for the Stix coil antenna. 


\subsection{Capacitive Antenna}

The Capacitive antenna consists of two aluminum semi-cylinders on each side of the plasma chamber (Figure 10). The plates were placed in parallel with a large inductor to match the RF source to the antenna circuit. The plates produce an oscillating electric field with radial and azimuthal components at the ion cyclotron frequency. The objective was to accelerate the ions in phase with their gyro orbit, similar to the $\mathrm{m}=0$ and Stix coil antennas. However, no heating was observed with this antenna. As with the $\mathrm{m}=0$ and Stix coils, the high ion collision frequency prevents coherent ion motion in the electric field.

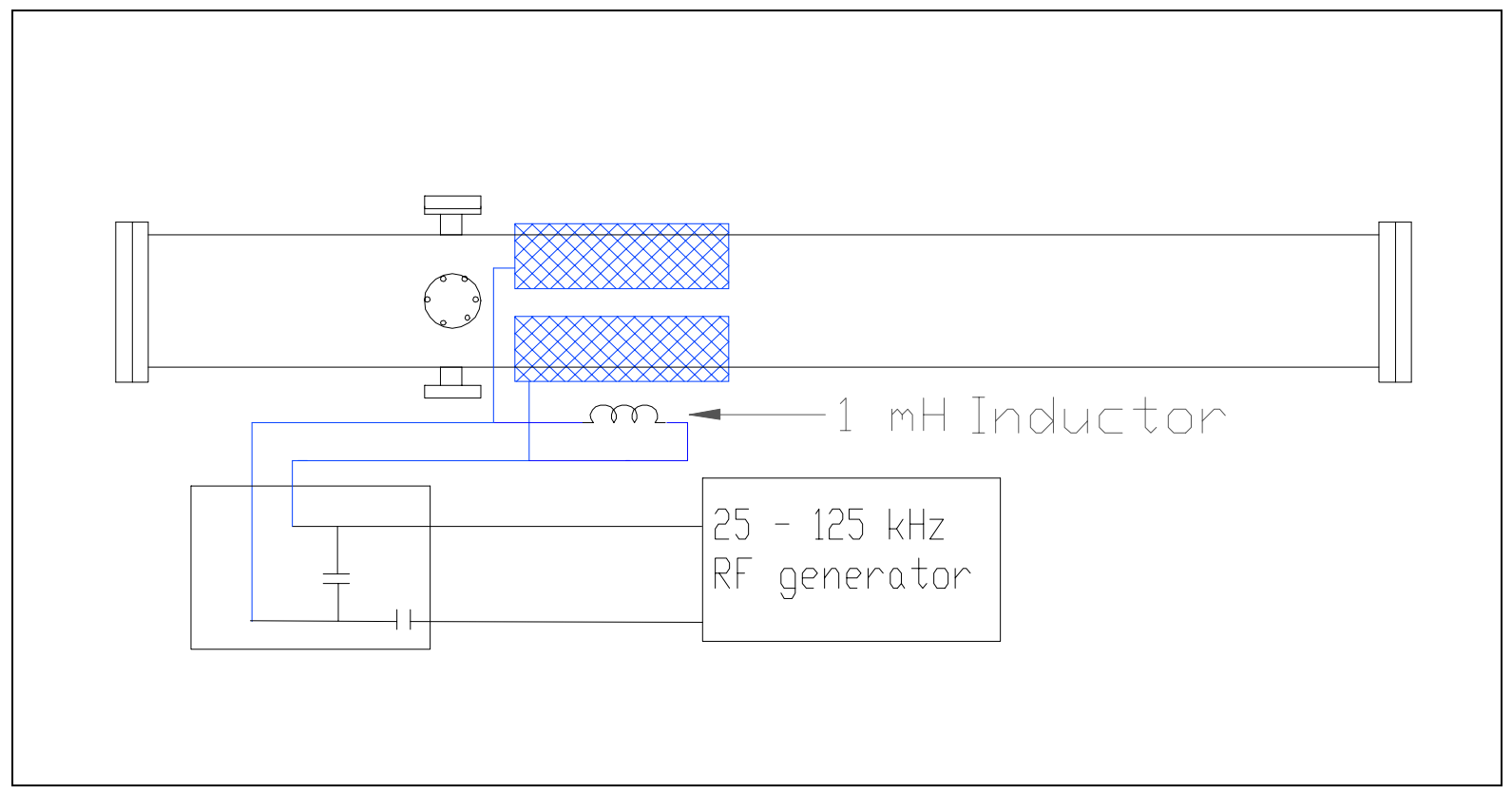

Figure 10. Capacitive antenna configuration. 


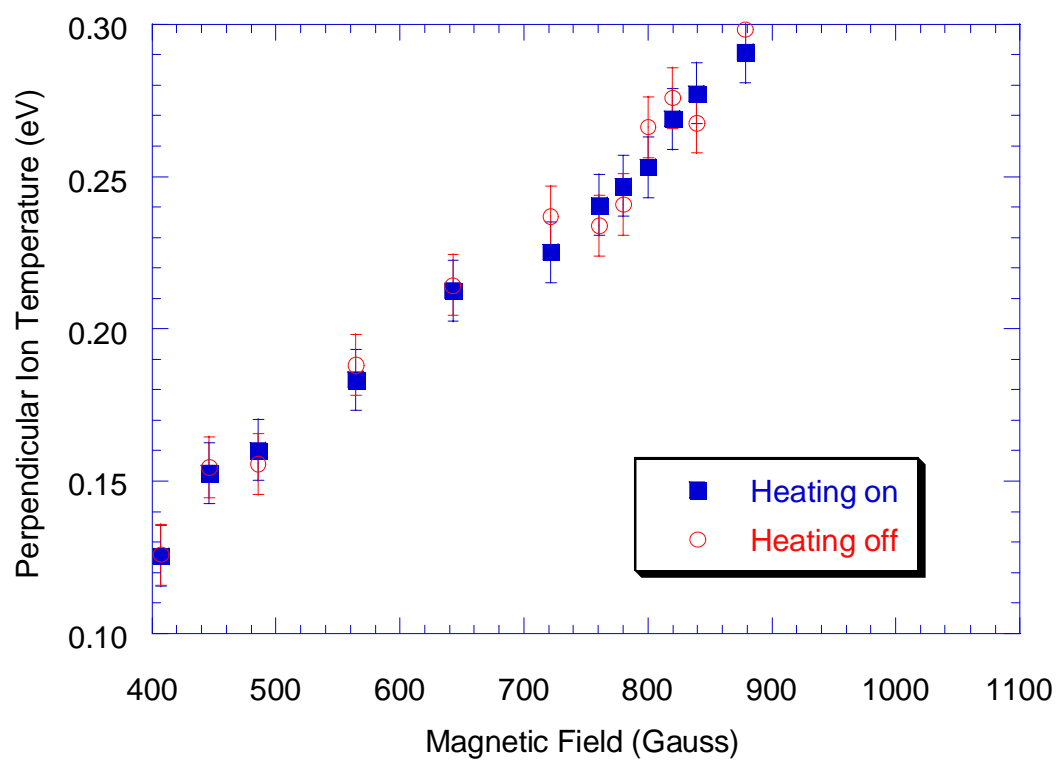

Figure 11. Perpendicular ion temperature versus magnetic field for heating circuit on and off for the capacitive antenna.

\subsection{Circular Transverse and Rectangular Transverse Inductive Antennas}

Ion heating experiments in magnetic mirror devices and tokamaks have used loop antennas [17] with the cylindrical axis of the antenna pointing towards the center of the plasma. These antennas heat the plasma through wave particle interactions. Based on those results, a Circular Transverse antenna (Figure 12a) consisting of two 36-turn coils of 12-gauge wire placed on opposite sides of the vacuum chamber was constructed. The coils create a small time dependent magnetic field transverse to the axial magnetic field. With this antenna, significant ion heating was observed (Figure 13). The heating efficiency versus $f / f_{\text {ci }}$ (heating frequency/ gyro frequency) data shown in Figure 14 indicate a peak heating efficiency of $32 \%$ at $f / f_{\mathrm{ci}} \approx 1.3$. Note that the heating is peaked at a unique frequency with a broad resonance. After the achievement of ion heating, a preliminary computational model of the ion motion suggested that a larger coil with more plasma coverage would increase the amount of ion heating, thus, the long, Rectangular, Transverse, antenna (RTA) was constructed. 


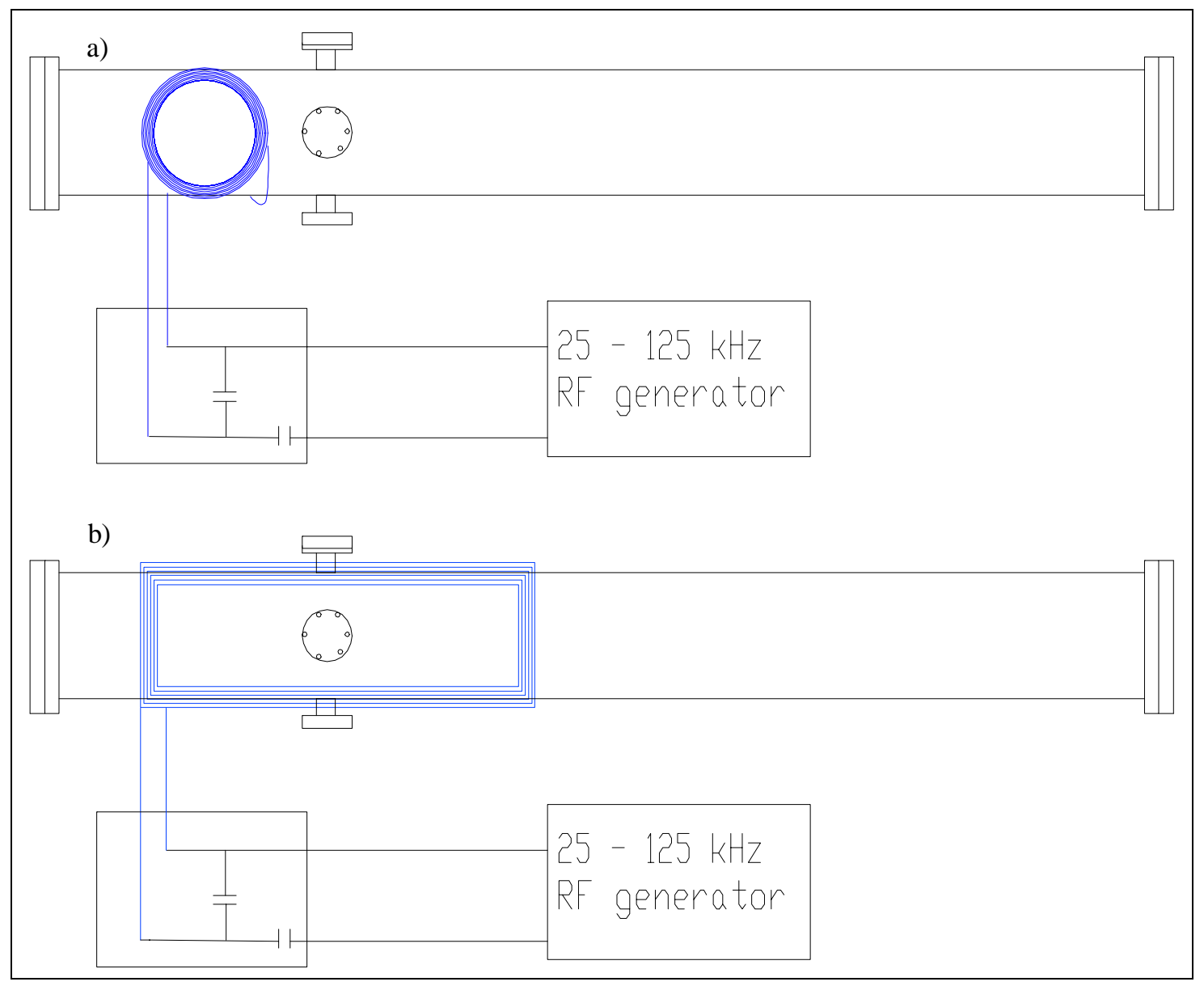

Figure 12. a) Circular Transverse antenna b) Rectangular Transverse antenna (only the near coil shown for each antenna)

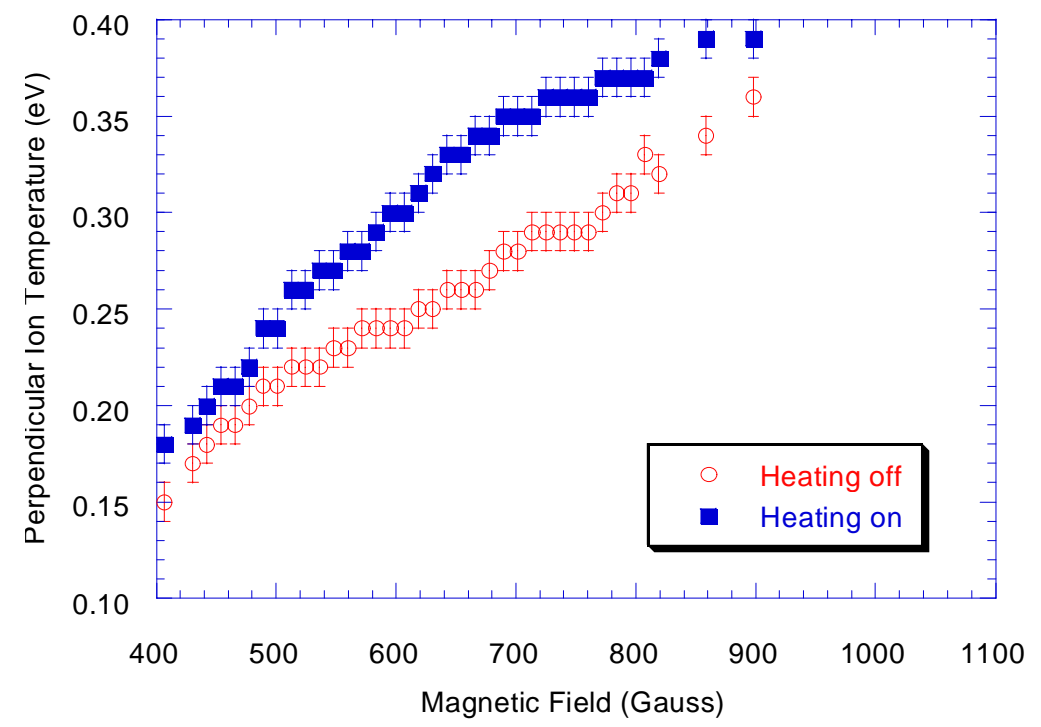

Figure 13. Perpendicular ion temperature verses magnetic for the circular transverse antenna. 


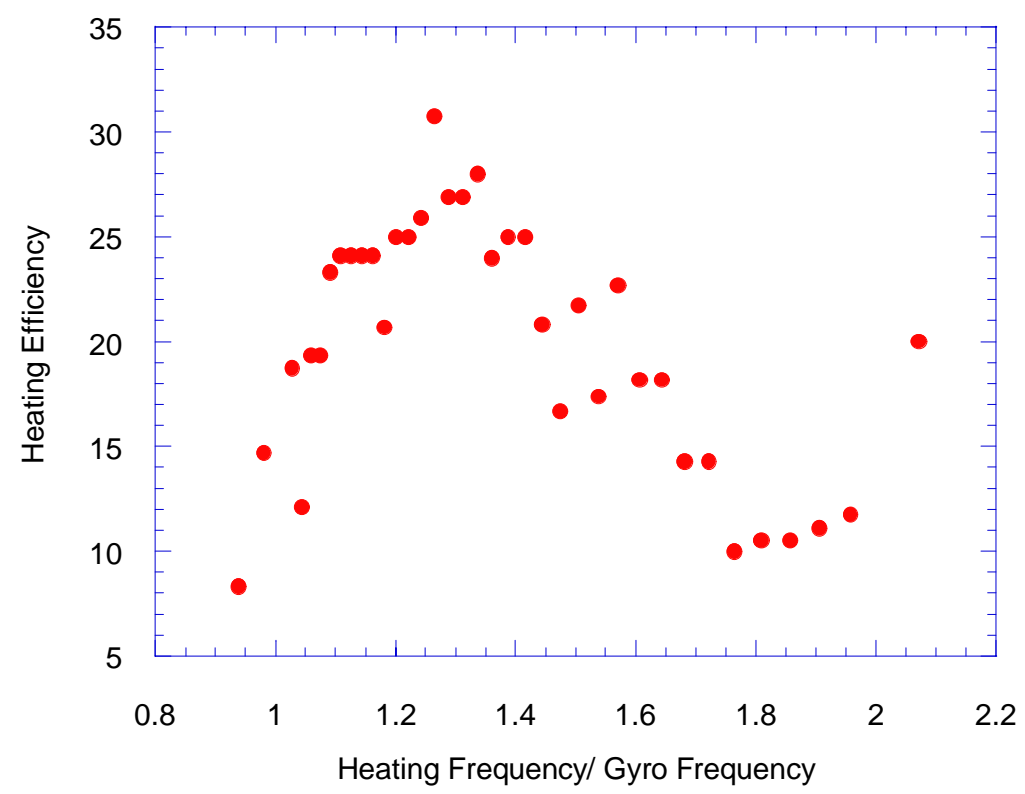

Figure 14. Heating efficiency for the circular transverse antenna versus heating frequency/ ion gyro frequency. The heating is broadly resonant at $f / f_{\mathrm{ci}} \approx 1.3$.

The long Rectangular Transverse antenna (RTA) (Figure 12b) consists of two 20 turn rectangular loops of 12-gauge wire, $55 \mathrm{~cm}$ long and $20 \mathrm{~cm}$ wide. This antenna creates a magnetic field of 25 Gauss for 27 amps over a plasma region about 3 times larger than the Circular Transverse antenna. With the RTA, the peak ion heating efficiency in the perpendicular direction was $80 \%$ for a neutral pressure of 3.0 mTorr (Figure 15). Because significant ion heating results were obtained with the RTA, parallel ion temperatures, as well as both perpendicular and parallel ion temperature measurements for a neutral pressure of 1.8 mTorr (Figure 16) were made. At a neutral pressure of 3.0 mTorr, the RTA produces a broad resonance centered at $f / f_{\mathrm{ci}} \approx 1.3$ with a peak heating efficiency of $80 \%$. At this pressure, the parallel ion temperature shows a resonance centered at $f / f_{\mathrm{ci}} \approx 1.2$. At the lower magnetic fields, the heating efficiency for the perpendicular ion temperatures is double that of the parallel heating efficiency. Such anisotropic ion heating is remarkable considering the ion collision frequency. 


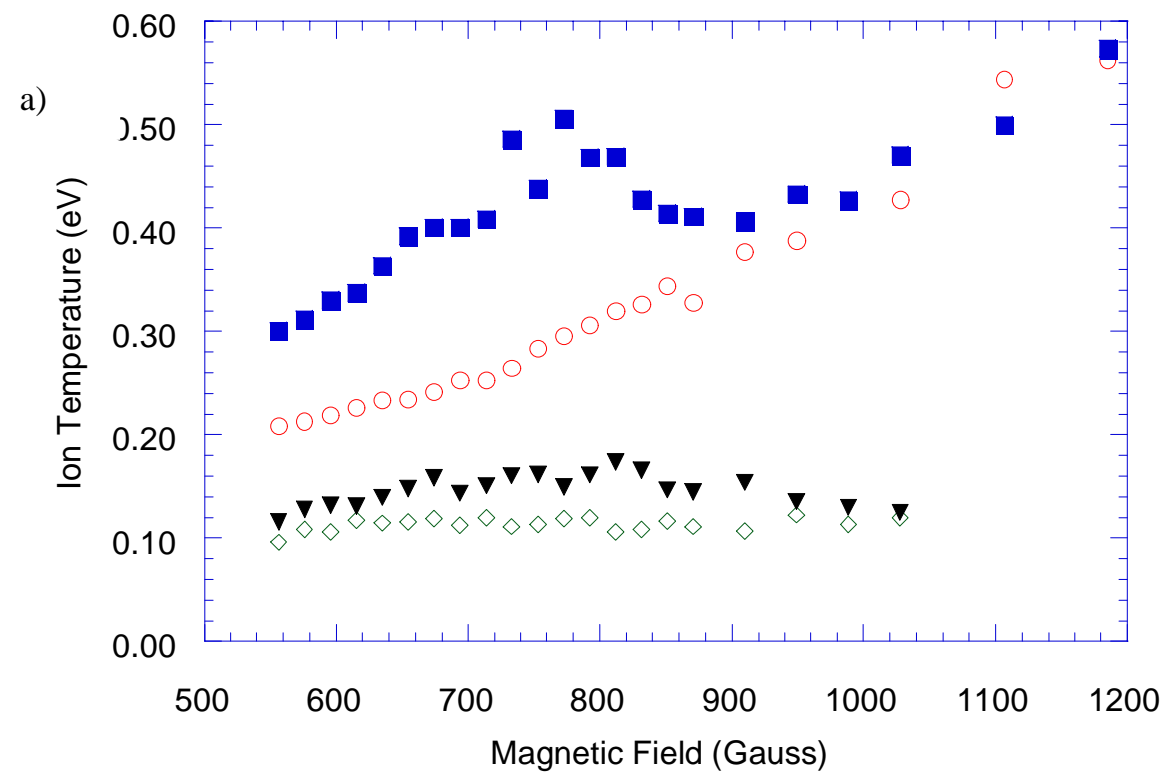

- Perpendicular Temperature - Heating off

- Perpendicular Temperature - Heating on

$\diamond \quad$ Parallel Temperature - Heating off

$\nabla$ Parallel Temperature - Heating on

b)

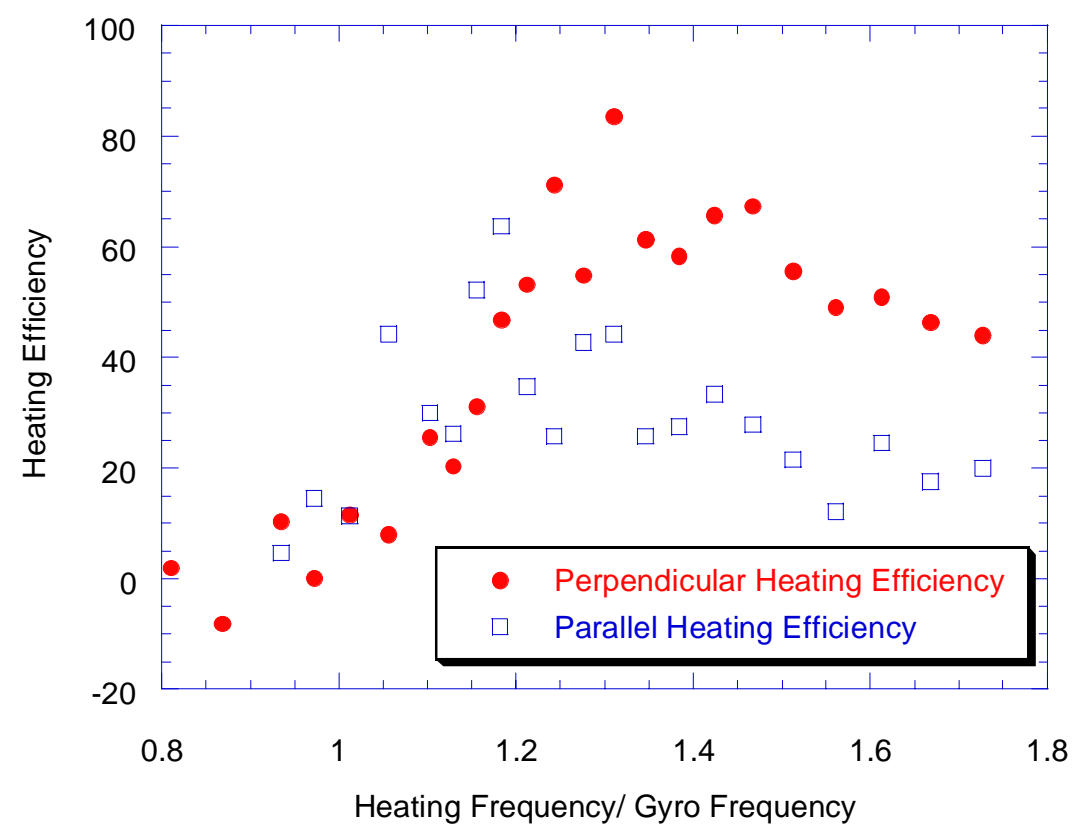

Figure 15. At a neutral pressure of 3.0 mTorr and heating power of $200 \mathrm{~W}$, a) the perpendicular and parallel ion temperatures with the heating circuit on and off versus the magnetic field strength. b) The heating efficiency for both the perpendicular and parallel directions. The heating is broadly resonant at $f / f_{\mathrm{ci}} \approx 1.3$ for the perpendicular temperature and $f / f_{\mathrm{ci}} \approx 1.2$ for the parallel temperature. 
a)

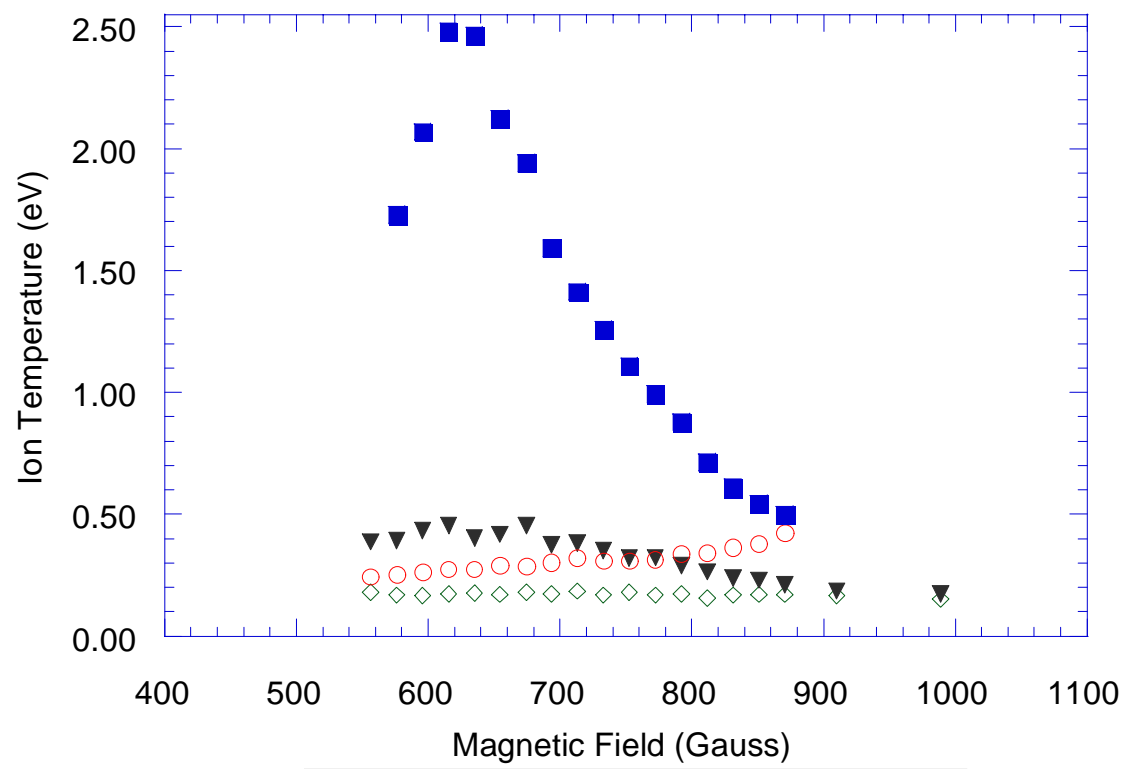

○ Perpendicular Temperature - Heating off

- Perpendicular Temperature - Heating on

$\diamond \quad$ Parallel Temperature - Heating off

$\nabla$ Parallel Temperature - Heating on

b)

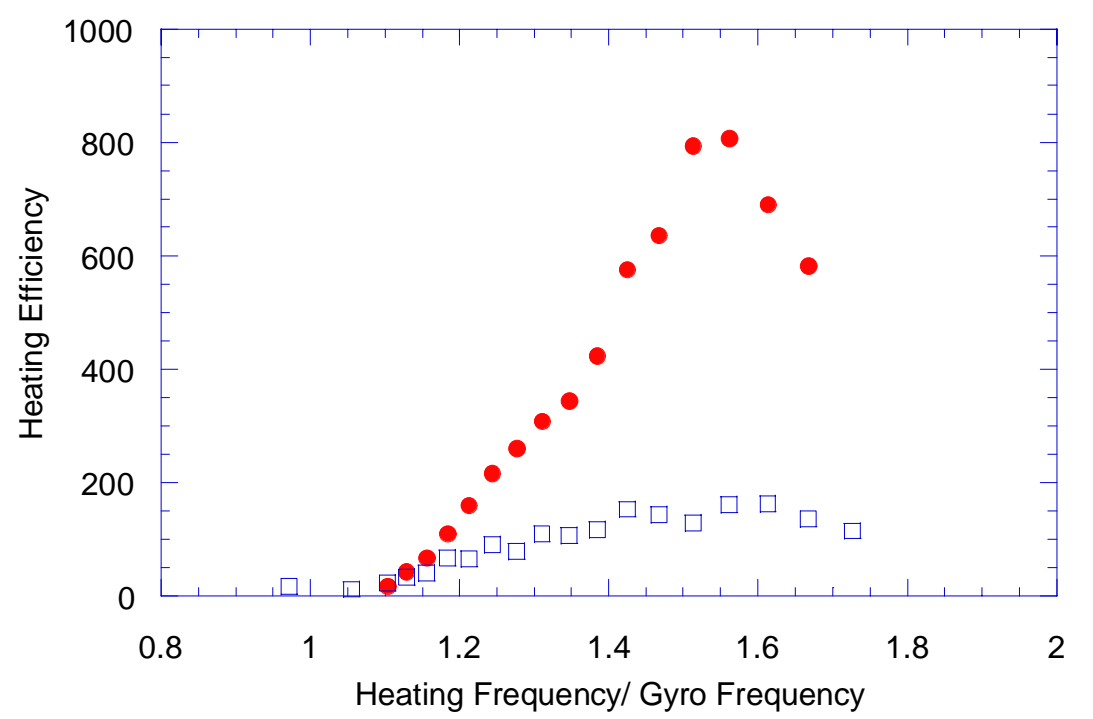

- Perpendicular Heating Efficiency

$\square \quad$ Parallel Heating Efficiency

Figure 16. At a neutral pressure of $1.5 \mathrm{mT}$ Torr and heating power of $200 \mathrm{~W}$, a) the perpendicular and parallel ion temperatures with the heating circuit on and off versus the magnetic field strength. b) The heating efficiency for both the perpendicular and parallel directions. The heating is resonant at $f / f_{\mathrm{ci}} \approx 1.5$ for the perpendicular temperature. 
At a neutral pressure of $1.5 \mathrm{mTorr}$, the peak perpendicular heating efficiency jumps to $800 \%$ and occurs at $f / f_{\text {ci }} \approx 1.5$. The resonance for the parallel heating efficiency is very broad and a clearly defined peak is not apparent. At this pressure, HELIX is in the inductive mode, not the helicon mode [1]. In the inductive mode, the density decreases by an order of magnitude, which lowers the collision frequency by an order of magnitude, $v_{i i}$ $\Omega_{i} \sim 4$. The perpendicular and parallel ion temperatures are not as collisionally coupled, and the ion heating is clearly anisotropic.

For both 3.0 mTorr and 1.5 mTorr, the RTA resonantly heats the ion at a specific $f / f_{\text {ci. }}$. The magnitude of the heating efficiency also depends on the antenna current. Figure 17 shows that for a neutral pressure of 3.0 mTorr and with the magnetic field set for the peak heating efficiency, the heating increases quartically as the current in the coil antenna is increased. The current in the coil antenna is directly proportional to the magnetic field generated by the coil antenna.

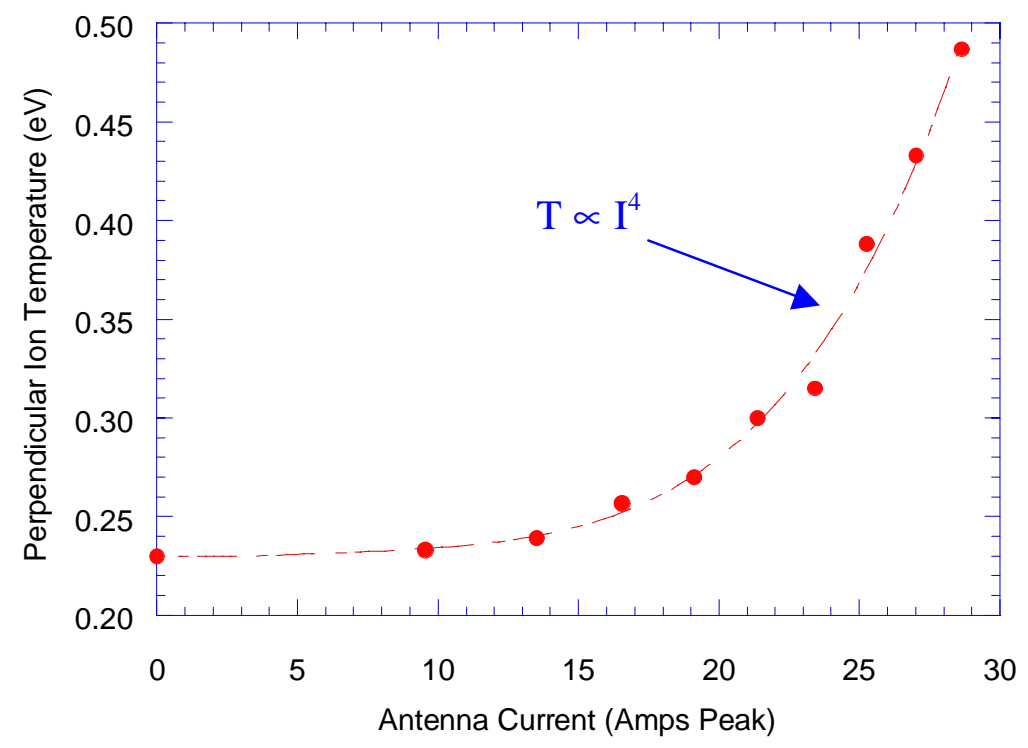

Figure17. Perpendicular ion temperature verses RTA current for a neutral pressure of $3.0 \mathrm{mTorr}$ and the magnetic field chosen for peak heating efficiency. The current in the antenna coil was directly measured to be 27 amps at 200 Watts heating power and the antenna circuit impedance was calculated. This impedance was then used to convert the heating power to current for this graph. 


\subsection{Transverse Inductive Plus Capacitive Antenna}

The transverse inductive plus capacitive antenna is a combination of both the circular transverse inductive antenna and the capacitive antenna (Figure 18). This configuration was attempted purely out of an experimentalist's trial and error curiosity. It was already known that the circular transverse antenna heated the ions. The aluminum plates were added to see if they had any enhancing effect. The heating results (Figure 19) showed no systematic variation in the heating efficiency with $f / f_{\mathrm{ci}}$. Compared to the circular transverse antenna by itself, the efficiency varies widely from larger to smaller values as $f / f_{\mathrm{ci}}$ is varied. Clearly the capacitive antenna modified the coupling of the transverse coil antenna to the plasma, but the resulting heating efficiency data did not lend itself to any obvious physical explanations.

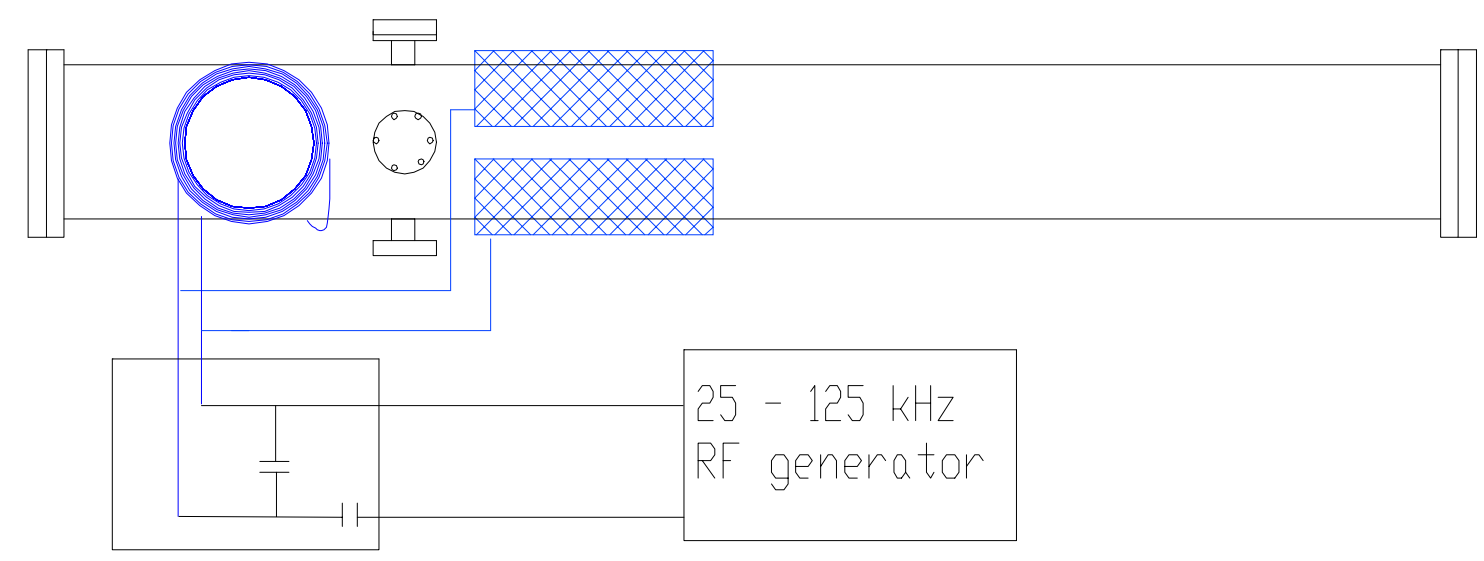

Figure 18. Transverse circular antenna plus the capacitive antenna 
a)

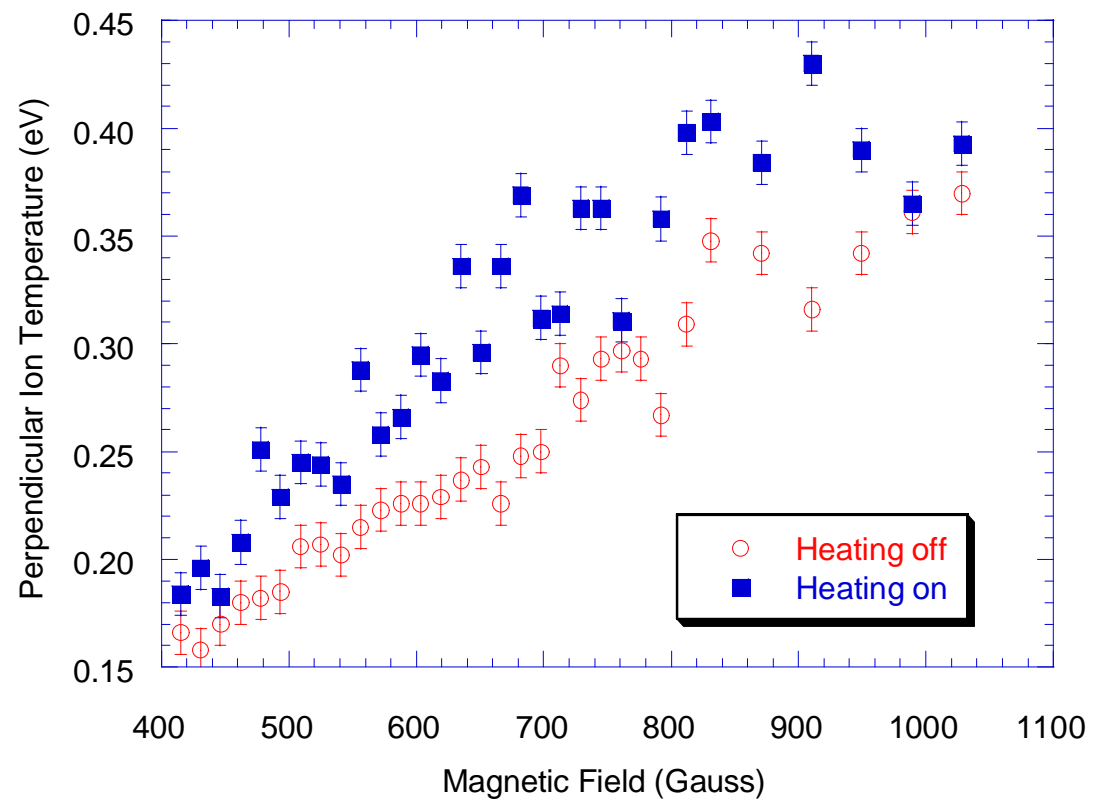

b)

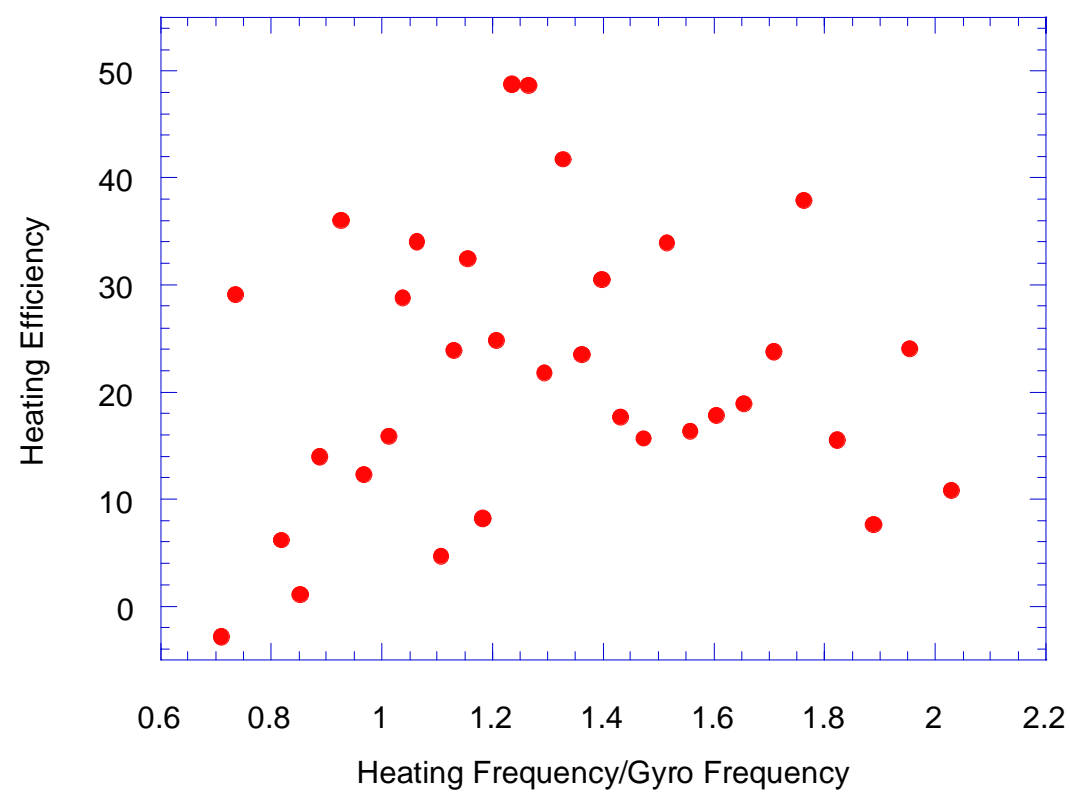

Figure 19. At a neutral pressure of $3.0 \mathrm{mTorr}$ a) the perpendicular ion temperature versus magnetic field for both the heating antenna circuit on and off. b) The heating efficiency verses the heating frequency/ ion gyro frequency for the circular transverse antenna plus the capacitive antenna. 


\section{Discussion}

There are three physical processes that could be responsible for the observed ion heating: electron-ion collisions, stochastic ion heating, and wave particle heating. In previous work, it was suggested that electron-ion collisions are responsible for the increase in ion temperature with increasing magnetic field in a helicon source [3]. Since the electron temperature is roughly 20 - 50 times larger than the ion temperature, electron-ion thermalization increases the ion temperature if the ion energy confinement time is longer than the electron-ion relaxation time. Scime et al. [3] hypothesized that increased perpendicular particle confinement due to larger magnetic fields preferentially increased the perpendicular ion temperature by increasing the number of electron-ion collisions an ion experiences.

However, electron-ion collisional heating is not a likely candidate for the resonant ion heating observed in these experiments. The data for the resonant heating experiments are not consistent with an electron-ion collisional heating mechanism. For electron-ion heating to be responsible, the low frequency RF would either have to increase the electron temperature or the ion energy confinement time. No increase in the electron temperature was observed. A simplistic single particle trajectory following code was developed to investigate the effect of the transverse magnetic field on the ion motion. The additional magnetic field could lead to trapping of the ions in the antenna region for particular initial conditions, but the relatively high ion collision frequency prohibits the ions from following such trajectories. The ion mean free path for the $3.0 \mathrm{mTorr}$ case is $0.01 \mathrm{~cm}$, much smaller that needed to follow the intricate trapping trajectories observed in the simulation.

Stochastic ion heating is a nonlinear heating mechanism that has been observed in a variety of experiments [18]. Essentially, stochastic heating arises when the acceleration experienced by a particle during its gyro motion is large enough that its change in average velocity during the gyro motion is significant, i.e., $(d v / d x) / \Omega_{i}>1$. In other words, the forces on the particle have to be evaluated at every point along its path and not at the guiding enter. For ion motion in a wave that is propagating perpendicular to the magnetic 
field and is purely longitudinal, e. g. near a resonant layer, the ion motion can be described by [9]

$$
\begin{gathered}
\dot{x}=\Omega_{i} \dot{y}+\frac{e}{m_{i}} E_{o} \cos (k x-\omega t) \\
\ddot{y}=-\Omega_{i} \dot{x}
\end{gathered}
$$

with an appropriate choice of constants and scales, (1) becomes

$$
\begin{gathered}
\ddot{x+x}=\alpha \cos (x-v t) \\
v=\omega / \Omega_{i} \\
\alpha=E k / B_{o} \Omega_{i}
\end{gathered}
$$

where $E_{o}=$ wave amplitude, $k=$ wave number, $B_{o}=$ local magnetic field strength, $\Omega_{i}=$ ion gyro frequency, $\omega=$ wave frequency. Stochastic motion of the particle in phase space (heating) occurs above some threshold value of $\alpha$ [19].

With stochastic heating, one would expect to see the ion heating increase once the stochastic threshold for $\alpha$ is reached. Since $\alpha$ is inversely proportional to the square of the magnetic field, $B_{o} \Omega_{i}$, stochastic heating is more likely to occur with smaller magnetic fields. As the magnetic field increases, the stochastic heating should decrease. These data show a peak heating for a specific magnetic field strength as the magnetic field increases, inconsistent with a stochastic heating mechanism.

The most probable candidate for the resonant ion heating is wave-particle heating. When a wave is excited in a plasma by an external driver, the wave can transfer energy to the particles in the plasma whose velocities are near the phase velocity of the wave. This allows the external energy source to inject energy to the plasma, and increase the kinetic energy of those particles. In this experiment, the ions are relatively cold and a wave with a small phase velocity is required. Near a resonant frequency of a plasma, the phase velocity of a wave goes to zero as the wavelength goes to zero. In fact, this is the definition of a plasma resonance [20]. Since the data (Figure 15b \& 16b) suggest that such a resonant process occurs for $1.2 \Omega_{i}<\omega<1.5 \Omega_{i}$ depending on the plasma and/or the neutral density, 
the obvious candidates for wave-particle heating should have a frequency or harmonic near the ion cyclotron frequency.

One electromagnetic wave that meets this criterion is the ion Bernstein wave [21]. It has been shown by Abe et al. [22] and Porkolab [23], that ion Bernstein waves result in strong anisotropic, $\Delta T_{\perp}>\Delta T_{\| /}$, ion heating when they are driven at $\omega=3 / 2 \Omega_{c i}$. The ion Bernstein wave is a hot plasma phenomena and does not appear in the cold plasma dielectric tensor [12]. A nonlinear process mode converts the ion Bernstein wave into an ion cyclotron wave at the third harmonic of the cyclotron frequency. Abe et al. [22] modeled this process using time dependent Hamiltonian perturbation theory for single particles in a slab geometry under the influence of an electrostatic wave, $\phi_{o} \cos \left(k_{\perp} x+k_{\mathbb{R}}-\omega t\right)$, and a background magnetic field. The equation of motion for the particles in the $x$ direction is

$$
\ddot{x}+\Omega_{c i}^{2} x=(q / m) k_{\perp} \phi_{o} \cos \left(k_{\perp} x-\omega_{d} t\right)
$$

where the symbols have there usual meaning. $\omega_{d}=\omega-k_{\|} v_{z o}$, where $v_{z o}$ is the initial $z$ velocity. Their results show resonant heating at $1.5 \Omega_{c i}$. The agreement between the heating data for the low pressure Rectangular Transverse antenna and Abe et al. [22] prediction is striking (Figure 20).

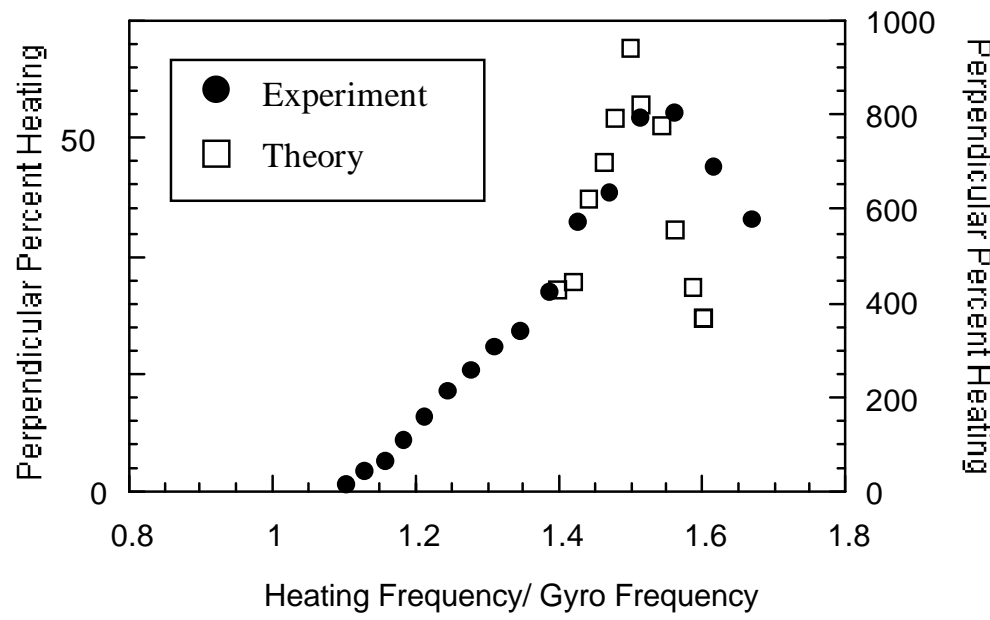

Figure 20. Theoretical results of Abe et al. [22] for ion Bernstien wave heating. This graph shows the heating efficiency multiplied by $k_{b} / N$ vs. the heating frequency / gyro frequency. 
The excitation of an ion Bernstein wave requires an electric field component parallel to the background magnetic field. With an appropriate choice of axis for a loop antenna (Figure 12b), an electric field component along the magnetic field can be created. At the correct frequency, this can excite an ion Bernstein wave. This is the type of antenna used in this experiment [17].

A estimate of the ion Bernstein wavelength can be obtained from an equation given by Wurden et al. [24]:

$$
\lambda_{\perp}=\left(\frac{T_{i}}{m_{i}}\right)^{1 / 2} \frac{1}{f}\left\{\beta /\left[4\left(\Omega_{i}^{2} / \omega^{2}\right)-1\right]\right\}^{1 / 2}
$$

where $T_{i}$ is the ion temperature, $m_{i}$ is the ion mass, $f$ is the frequency, and $\Omega_{i}$ is the ion cyclotron frequency. For our argon plasma, $T_{i} \sim 0.3 \mathrm{eV}, f \sim 35 \mathrm{kHz}$, and $\Omega_{i} / \omega \sim 0.66$, the wavelength, $\lambda_{\perp} \approx 5 \mathrm{~cm}$, is comparable to the circumference of the blue core portion of our plasma $[1,3]$. The agreement between the theoretical and computational prediction with the experimental results strongly support the conclusion that an ion Bernstein wave is responsible for the ion heating. Direct measurements of the wavelength and comparison to the dispersion relationship for an ion Bernstein wave would provide unequivocal evidence of ion Bernstein Wave heating.

\section{Conclusions}

These experiments demonstrate that anisotropic ion heating using low frequency $\mathrm{RF}$ is possible in a helicon source. At $3.0 \mathrm{mTorr}$, the resonance is broad, $\Delta f / f \approx .3$, and is centered about $f / f_{c i} \approx 1.3-1.4$ with a peak ion heating efficiency of $80 \%$. The associated heating anisotropy, perpendicular heating efficiency divided by the parallel heating efficiency, at 3.0 mTorr is approximately 2. At $1.5 \mathrm{mTorr}$, the heating resonance is narrower, $\Delta f / f \approx .2$, and is centered about $f / f_{c i} \approx 1.5$ with a peak heating efficiency of $800 \%$. The associated heating anisotropy at $1.5 \mathrm{mTorr}$ neutral pressure is approximately 5. From these data, the antenna geometry, and theoretical predictions, the heating appears to be a result of the absorption of ion-Bernstein-waves through nonlinear ion Landau damping. 


\section{Acknowledgements}

I would like to especially thank my advisor Professor Earl Scime for his assistance and his temperament, which enabled him to put up with me through this learning experience. I would also like to thank Paul Keiter, Dr. Robert Boivin, Matt Balkey, and Dr. Mike Zintl for their help with this project and many useful conversation about the physics envolved. I want to give a big thanks to my parents, my sisters, and the rest of my family for their support.

I would like to acknowledge and thank Professor Mark Koepke for the installation of the laser system instrumental to this project. Furthermore, I want to thank Professor Mark Koepke, Professor Tim Good, Dr. Mike Zintl, and Professor Earl Scime for their contribution in the design of the optical system and support tuning the laser.

I would like to acknowledge Professor Mike Mauel at Columbia University for his suggestion of the transverse antenna coils. I would also like to thank Mr. Tom Lovell for the capacitors used in the antenna matching circuit and his help in the design and understanding of the matching circuit. I want to thank Professor Fred Skiff at the University of Iowa for very useful information concerning ion Berstein waves.

This work has been supported by the Department of Energy and the National Science Foundation. 


\section{References}

[1] P. A. Keiter, E. E. Scime, and M. M. Balkey Phys. Plasmas 42741 (1997)

[2] S. P. Gary Space Plasma Instabilities (New York: Cambridge 1993)

[3] E. E. Scime, P. A. Keiter, M. W. Zintl, M. M. Balkey, J. L. Kline, and M. E. Koepke Plasma Sources Science and Technology, 7186 (1998)

[4] B. B. Kadomtsev Tokamak Plasma: A Complex Physical System (IOP Publishing, Bristol, Philadelphia 1992)

[5] S. N. Golovato, K. Brau, J. Casey, J. Coleman, M. J. Gerver, W. Guss, G. Hallock, S. Horn, J. Irby, R. Kumazawa, J. Kesner, B. Lane, J. Machuzak, T. Moran, R. Myer, R. S. Post, E. Sevillano, D. K. Smith, J. D. Sullivan, R. Tori, L. Wang, Y. Yasaka, X. Z. Yao, and J. Zielinski. Phys of Fluids 313744 (1988)

[6] D. J. Rose, M. Clark Jr. Plasmas and Controlled Fusion (John Wiley \& Sons, The MIT Press, New York, London 1961)

[7] T. N. Good, H. R. Thompson Jr., and N Rynn Phys. of Fluids 311237 (1988)

[8] N. Rynn, D. R. Dakin, D. L. Correll, and G. Benford Phys. Rev. Lett. 33765 (1974)

[9] R. A. Cairns Radiofrequency Heating of Plasmas (Adam Hilger, Bristol, Philadelphia, and New York 1991)

[10] W. M. Stacey jr. Fusion: An Introduction to the Physics and Technology of Magnetic Confinement Fusion (John Wiley \& Sons, New York 1984)

[11] B. Grek and M. Porkolab Phys. Rev. Lett. 30836 (1973)

[12] M. Ono Physics of Fluids B 5241 (1993)

[13] M. Light, I. D. Sudit, F. F. Chen, and D. Arnush Phys. Plasmas 24094 (1995)

[14] D. N. Hill, S. Fornaca, and M. G. Wickham Rev. Sci. Instrum. 54309 (1983)

[15] D. A. Edrich, Ph. D. Thesis, University of California Irvine (1995)

[16] T. H. Stix and W. R. Palladino Phys. of Fluids 1446 (1958)

[17] M. Ono, T. Watari, R. Ando, J. Fujita, Y. Hirokura, K. Ida, E. Kako, K. Kawahata, Y. Kawasumi, K. Matsuoka, A. Nishizawa, N. Noda, I. Ogawa, K. Ohkubo, M. Okamoto, K. Sato, S. Tanahashi, Y. Taniguchi, T. Tetsuka, K. Toi, and K. Yamazaki Phys. Rev. Lett. 542339 (1985)

[18] J. M. McChesney, P. M. Bellan, and R. A. Stern Phys. of Fluids B 33363 (1991)

[19] C. F. F. Karney and A. Bers Phys. Rev. Lett. 39550 (1977)

[20] F. F. Chen Introduction to Plasma Physics and Controlled Fusion (Plenum Press, New York, London, (1984)

[21] I. B. Bernstein Phys. Rev. Lett. 10 (1958) 
[22] H. Abe, H. Okada, R. Itatani, M. Ono, and H. Okuda Phys. Rev. Lett. 531153 (1984)

[23] M. Porkolab Physical Review Letters 54434 (1985)

[24] G. A. Wurden, M. Ono, and K. L. Wong Phys. Rev. A 262297 (1982) 